\title{
Learning with small amount of data on various task
}

\author{
Atsushi Kondo, Tu Tran, Pratik Sutar
}

\begin{abstract}
As a student, I am learning knowledge with the help of teachers and the teacher play a crucial role in our life. A wonderful instructor is able to teach a student with appropriate teaching materials. Therefore, in this project, I explore a teaching strategy called learning to teach (L2T) in which a teacher model could provide high-quality training samples to a student model. However, one major problem of L2T is that the teacher model will only select a subset of the training dataset as the final training data for the student. A learning to teach small-data learning strategy (L2TSDL) is proposed to solve this problem. In this strategy, the teacher model will calculate the importance score for every training sample and help student to make use of all training samples.

To demonstrate the advantage of the proposed approach over L2T, I take the training of different deep neural networks (DNN) on image classification task as an example and show that L2TSDL could achieve good performance on both large and small dataset.
\end{abstract}

\section{An Introduction}

An advanced education system plays a crucial role in our society and the goal of teaching is to equip people with necessary skills which could help people further understand this world. In general, students should pay more attention to the learning materials which could help them to learn knowledge better and faster. In reality, great teachers are able to select "good" materials for their students and help them learn new knowledge as quickly as possible.

In the field of artificial intelligence, the training of a model, e.g., an image classifier, is very similar to the learning process of a student. This model does not know the knowledge (e.g., what is a "dog") at first but after training (studying) by some training samples (teaching materials), it could learn the representation of different items and detect them. However, it is clear to find that there is no "teacher" in this process, which means that there is not an agent that could tell this model how to learn the training samples as effectively and efficiently as possible.

However, nowadays, researchers would like to pay more attention to the "student" itself. In contrast, the number of attempts on incorporating teachers into training process is limited. Some work [28, 29. 19] investigate how to identify the smallest training set to motivate machine learning algorithms to a pre-defined oracle model. Also, Self-paced learning (SPL) [16, 17] and Curriculum learning (CL) [2, 5] study the ordering of the training data in a from-easy-to-hard order. However, these models and attempts are based either on task-specific heuristic rules or a strong assumption of a pre-known golden model existence. Therefore, these models could not change their training strategy according to different training samples and the amount of the data. Furthermore, these models could not evolve themselves according to the feedback from the student model. For example, if the training loss of the student model does not decrease, the "teacher" should change its strategy to teach the student.

Recently, "learning to teach (L2T)" framework [27], automating the teaching process using an endto-end trainable method, has been proposed. In this framework, there are two intelligent modules: 1). 
a student module to learn an artificial intelligent task, e.g., image classification; 2). a teacher module to select the suitable data from the training samples for the student model.

In the training process, the teacher learns how to choose appropriate data for the student model and the student performs its training process based on the data selected by the teacher. According to the student performance (accuracy on the held-out dataset), the teacher will change its strategy on data selection via a policy gradient algorithm [25]. The advantage of this method is that this interactive process is end-to-end and after the teacher is trained, it could be applied to teach students in similar scenarios (e.g., image classification) without re-training.

However, in this framework, only the data which the teacher thinks is useful is selected for the student training and the other samples will be ignored. It is reasonable to use this strategy when we have a large amount of data. But if only a small amount of training data is available, it will be relatively difficult to train a student model successfully with just part of the training data.

Therefore, in this project, I would like to extend L2T framework to the small-data learning scenario. In more details, instead of just deciding whether to keep or ignore a training instance, the teacher could judge the importance level of each training instance. In another word, for informative training samples, the teacher will assign a large weight on them and make the student pay more attention to them. In order to verify the effectiveness of the proposed method, an image classification task on two standard datasets, i.e., MNIST and CIFAR-10 are explored.

\section{Related Works}

Machine teaching [28, 29] tries to find a minimal data set which could be used to learn an oracle model for a student model. Furthermore, the teaching dimension of several students has been defined [18] and machine teaching is extended to iterative setting [19]. However, since these models are based on the assumption of oracle existence, they have only been applied to a few fields, such as human-computer interaction [21, 9], machine translation [14, 13, 26], speech [10, 15, 11, 12]

Hardness based methods is another important branch of the machine teaching, which do not assume that there is an oracle model. Specifically, hardness based methods find that it would be easy to optimize the learning process in the order of from-easy-to-hard. Therefore, the goal of hardness based methods is to measure the hardness of training samples. In particular, curriculum learning $([2,5,24]$ tries to measure the hardness by heuristic understanding of data. In contrast to the curriculum learning, self-paced learning $([16,17,22]$ measures the hardness of samples by the loss of them.

Another trend of machine learning recently is from simple learning to advanced learning. For example, meta learning. [7, 1]. tries to explore the abilities of automatic learning through transferring generic knowledge learned from meta tasks and recently has been widely applied in many machine learning tasks, such as few-shot learning [20, 4].

\section{Problem Definition}

In this project, I only focus on the supervised learning.

In supervised learning scenario, there is an input space $\mathbf{X}$ and an output space $\mathbf{Y}$. Given a sample drawn from the input space with a distribution $P(x)$, a label is obtained according to a conditional distribution $P(y \mid x)$. Note that distributions $P(x)$ and $P(y \mid x)$ are unknown but fixed. The goal of the supervised learning is to learn a function $f(x)$ with parameters $\theta$ which could fit the real distribution $P(y \mid x)$ as well as possible. In another word, given a sample $x, f(x)$ tries to predict the golden label $y$ of the sample as accurate as possible. In order to measure the performance of the function $f(x)$ during the training process, we often design a loss function $L(f(x), y)$ which could calculate the distance between the predicted label and the golden label.

Since real source distribution $P(x, y)$ is unknown and the learning process of supervised learning is based on the training data $D=\left\{x_{i}, y_{i}\right\}_{i=1}^{n}$, the actual learning process corresponds to the following equation:

$$
\phi^{*}=\operatorname{argmin}_{\phi} \sum_{(x, y) \in D} L\left(y, f_{w}(x)\right)
$$


Therefore, the quality of the training data is crucial to the learning process. If the distribution of the training dataset is different from the real distribution, it will be very hard for any learning algorithm to obtain an accurate and robust $f(x)$. Note that in the learning to teach framework, we also call the $f(x)$ as the student model.

In order to make the distribution of the training data as similar as the truth distribution $P(x, y)$, it is necessary to incorporate a teacher model, which could provide the student model with the appropriate input, into the learning process. Specifically, in this project, there are two options to decide whether a training sample is appropriate or not.

- The teacher is able to remove training samples which are different from the truth distribution. Therefore, after data selection, the final training dataset is the subset of the original one. This method will be described as L2T in this project.

- The teacher will measure the importance score of every training sample. Intuitively, if a training sample is drawn from the real distribution $P(x, y)$, a good teacher will assign a higher score to it so that the student model will pay more attention to this sample. This method will be described as L2TSDL in this project.

\section{Method}

In this project, I would like to focus on the image classification task with three models.

No Teaching (NoTeach). In this model, I will use the tradition machine learning training process to build an image classier, which will be the baseline. Compared with L2T and L2TSDL framework, there is no teacher but just a student.

Learning to Teach (L2T). In this model, I will follow the method used in [27] which is declared in the following:

$S$ is a set of states. The state $s_{t} \in S$ at each time step $t$ represents the information available to the teacher model. $s_{t}$ is typically constructed from the current student model $f_{t}$ and current mini-batch samples $D_{t}$. At the $t$-th step, given the state $s_{t}$, the teacher model takes an action $a_{t} \in A$. $a_{t}$ here is a set of training data selected from the mini-batch samples $D_{t} . \phi_{\theta}: S \Rightarrow A$ is the policy with parameter $\theta$ employed by the teacher model to generate its action: $\phi_{\theta}\left(s_{t}\right)=a_{t}$. When without confusion, I also call $\phi_{\theta}$ the teacher model. The student model takes $a_{t}$ as input and outputs a function $f_{t+1}$, by using conventional machine learning technologies.

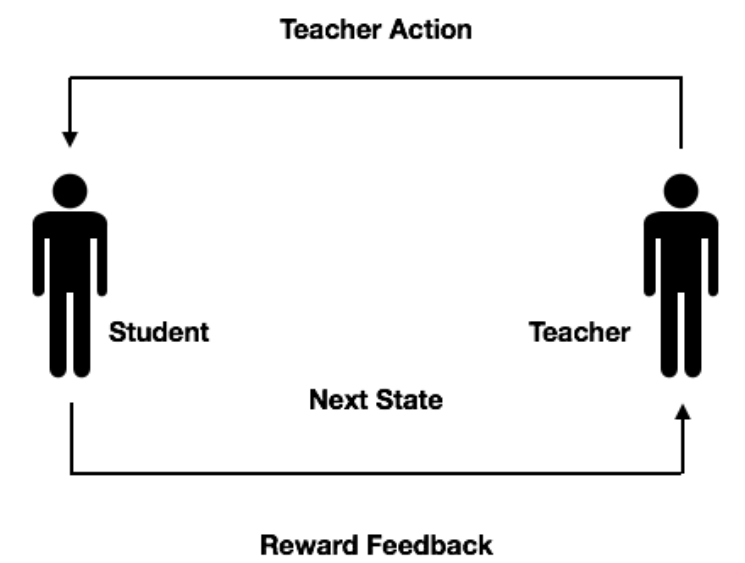

Figure 1: Learning to teach framework: the interactive process between a teacher and a student.

In L2T, reinforcement learning (RL) is employed to complete the interaction between a student model and a teacher model. In this case, the teacher model $\phi_{\theta}$ acts as the policy interacting with the environment, which is represented by $S$. After seeing the teaching action $a_{t}$, the student model updates itself based on $a_{t}$, changes the environment to $s_{t+1}$ and then provides a reward $r_{t}$ to the teacher model. The reward indicates how good the current student model $f_{t}$ is, e.g., the accuracy 
on the validation set. The teacher model then updates its own parameters $\phi_{\theta}$ to maximize the accumulated reward. Such an interactive process between the teacher model and the student model is illustrated in Fig. 1. The interaction process stops when the student model gets converged, forming one episode of the teacher model training.

Learning to Teach small data learning (L2TSDL). Since in L2T framework, for each of the training samples, the teacher will decide whether it will be a part of the input for the student or not. It is practical if we have a large amount of training data, however, if the amount of the training data is limited, it is unreasonable to further ignore some data. In contrast, it will be better if we could let the teacher evaluates the importance of training samples and guide the student to pay more attention to samples with higher importance. Although the training process of L2TSDL is similar to that of L2T, there is a major difference between them. In L2T, the goal of the teacher is to select a mini-batch from original training samples and feed it to the student model but in L2TSDL, the teacher aims to evaluate the importance difference among training samples.

The reason why I choose reinforcement learning method is that there is no golden label (importance of every training samples in L2TSDL and decisions on keeping or ignoring a certain training instance in L2T) for the teacher model. Therefore, it is reasonable to use the reward sent from the student model as the objective to optimize the teacher.

\section{Interactive Process of Teacher and Student via Reinforcement Learning for L2TSDL}

The state $S$ corresponds to the mini-batch data and the current version of the student model: $s_{t}=$ $\left(D_{t}, f_{t}\right)$ where $s_{t}$ is the state at the $t$-th time step, $D_{t}$ is the mini-batch arrived at the time step $t$ and $f_{t}$ is the state of the student at $t$-th time step. The teacher's action $a$ is denoted by $a=\left\{a_{m}\right\}_{m=1}^{M}$ where $M$ is the batch-size and $a_{m} \in[0,1]$ indicates the importance score of the $m$-th sample in $D_{t}$. In general, more informative training samples should be assigned a higher action score. The reward in L2TSDL is related to the speed of the student training. In more details, the reward $r$ is set as the terminal reward, i.e., $r_{t}=0, \forall t<T$ where $T$ is the last time step in an episode. To achieve this, an accuracy threshold $\tau \in[0,1]$ is set and the first mini-batch index $i$ in which the accuracy on the valid set exceeds $\tau$ is recorded, then we set reward for this episode as $r=-\log \left(i / T_{\max }\right)$ where $T_{\max }$ is a pre-defined maximum iteration number for the student model.

The teacher samples its action $a_{t}$ via policy $\phi_{\theta}(a \mid s)$. The objective function of the teacher model is maximizing the expected reward : $E_{\phi_{\theta}}[R(s, a)]$ where $R(s, a)$ is the state-action value function. However, the $R(s, a)$ is non-differentiable with regard to $\theta$. REINFORCE [25] algorithm is employed to optimize the teacher via the gradient: $\nabla_{\theta}=\sum_{1}^{T} E_{\phi_{\theta}\left(a_{t} \mid s_{t}\right)}\left[\nabla_{\theta} \log \phi_{\theta}\left(a_{t} \mid s_{t}\right) R\left(s_{t}, a_{t}\right)\right]$, where $R\left(s_{t}, a_{t}\right)$ is the reward at $t$-th time step. Since the reward is terminal reward, the final result is: $\nabla_{\theta} \approx \sum_{1}^{T} \nabla_{\theta} \log \phi_{\theta}\left(a_{t} \mid s_{t}\right) r_{T}$ where $r_{T}$ is the terminal reward mentioned above.

\section{State Feature for the teacher}

One important aspect in L2T and L2TSDL is to define the input to the teacher, i.e., extracting feature which could effectively and efficiently represent the current state [5]. Since the current state consists the arrived mini-batch and the current state of the student, the state feature contains three categories, i.e., data features, student model features and the combination of both data and the student model features. In more details:

- Data Features capture the information of data samples and these features are used in curriculum learning [2]. Here I use 1 of $|Y|$ to represent the label category, where $|Y|$ is the number of target classes in the task. For example, for MNIST, $|Y|$ is 10 because of ten digit classes.

- Student model features contain the current performance of the student model, such as average historical training loss and valid loss. Specifically, the first feature is the current iteration number, the second one is the averaged training loss until the current iteration and the last one is the best validation loss so far. All these three features are normalized by pre-defined numbers to make them into the interval $[0,1]$. 
- Combination features include both student and data status. Those features are able to measure how important the current training data is for the student model. There are also three features. The first one is the predicted probabilities for each class, the second one is the loss value on the data which is often used in the self-paced learning methods [16] and the third one is the margin value [3] on current training samples.

Based on these designed features, the dimension of the state feature vector is $25=10$ (Data Features) +3 (student model) +12 (combined features).

\section{Dataset}

MNIST. There are 70,000 handwritten digits images from 10 categories $(0,1, \ldots, 9)$. It has a training set of 60,000 examples and a test set of 10,000 examples. It is a subset of a larger set available from NIST. The digits have been size-normalized and centered in a fixed-size image. Furthermore, the black and white images from NIST were normalized to fit into a $28 \times 28$ pixel bounding box and anti-aliased, which introduced grayscale levels.

CIFAR-10. It is a popular dataset on image classification, which contains 60,000 images of size 32 $\times 32$ categorized into 10 classes. There are 50,000 images in the training set and 10,000 images in the test set.

\section{Experiments Setting}

I conduct experiments to compare the performance of different teaching strategies, i.e., NoTeach, L2T and L2TSDL on both large data set and small dataset. In order to make the comparison more general, the image classification task on MNIST and CIFAR-10 are adopted and the multi-layer perceptron (MLP) and convolutional neural networks (CNN) are employed to build image classifiers (student model). In more details, I employ the Resnet [6] as the CNN student model. Adam [8] is used to train the MLP student model and Momentum-SGD[23] is applied to train the CNN student model.

\section{Conclusion and future work}

Inspired by importance of teaching role in the real life, learning to teach (L2T) framework has been proposed to automate the teaching process. A teacher model in L2T could help a student model to select appropriate data samples to achieve better performance. However, L2T will only select a subset of training samples as the training dataset for the student model, which is acceptable if we have a large amount of training samples. However, if there is only a small amount of training samples, it will be harmful if we further throw away some data samples. Learning to teach small-data learning (L2TSDL) is proposed to solve this issue. Instead of just simply ignoring some samples, L2TSDL is able to assign an importance score to each training sample. Comprehensive experiments on the image classification task have demonstrated the effectiveness of the L2TSDL framework.

Few-shot learning is very popular recently and L2TSDL teaching strategy is very suitable to be applied in this scenario. Therefore, I could train a teacher on a large amount of dataset, such as ImageNet or Cifar-100, then this teacher could teach a student model on some few shot learning corpora, such as Omniglot. Since the teacher do not see any few shot learning data, this framework could be compared with other models fairly. 


\section{References}

[1] M. Andrychowicz, M. Denil, S. Gomez, M. W. Hoffman, D. Pfau, T. Schaul, and N. de Freitas. Learning to learn by gradient descent by gradient descent. In Advances in Neural Information Processing Systems, pages 3981-3989, 2016.

[2] Y. Bengio, J. Louradour, R. Collobert, and J. Weston. Curriculum learning. In Proceedings of the 26th annual international conference on machine learning, pages 41-48. ACM, 2009.

[3] C. Cortes, M. Mohri, and A. Rostamizadeh. Multi-class classification with maximum margin multiple kernel. In International Conference on Machine Learning, pages 46-54, 2013.

[4] C. Finn, P. Abbeel, and S. Levine. Model-agnostic meta-learning for fast adaptation of deep networks. arXiv preprint arXiv:1703.03400, 2017.

[5] A. Graves, M. G. Bellemare, J. Menick, R. Munos, and K. Kavukcuoglu. Automated curriculum learning for neural networks. arXiv preprint arXiv:1704.03003, 2017.

[6] K. He, X. Zhang, S. Ren, and J. Sun. Deep residual learning for image recognition. In Proceedings of the IEEE conference on computer vision and pattern recognition, pages 770 778, 2016.

[7] S. Hochreiter, A. S. Younger, and P. R. Conwell. Learning to learn using gradient descent. In International Conference on Artificial Neural Networks, pages 87-94. Springer, 2001.

[8] D. P. Kingma and J. Ba. Adam: A method for stochastic optimization. arXiv preprint arXiv:1412.6980, 2014.

[9] X. Kong, X. Chen, and E. Hovy. Decompressing knowledge graph representations for link prediction. arXiv preprint arXiv:1911.04053, 2019.

[10] X. Kong, J.-Y. Choi, and S. Shattuck-Hufnagel. Analysis of distinctive feature matching with random error generation in a lexical access system. The Journal of the Acoustical Society of America, 138(3):1780-1780, 2015.

[11] X. Kong, J.-Y. Choi, and S. Shattuck-Hufnagel. Evaluating automatic speech recognition systems in comparison with human perception results using distinctive feature measures. In 2017 IEEE International Conference on Acoustics, Speech and Signal Processing (ICASSP), pages 5810-5814. IEEE, 2017.

[12] X. Kong, P. Jyothi, and M. Hasegawa-Johnson. Performance improvement of probabilistic transcriptions with language-specific constraints. Procedia Computer Science, 81:30-36, 2016.

[13] X. Kong, Z. Tu, S. Shi, E. Hovy, and T. Zhang. Neural machine translation with adequacyoriented learning. In Proceedings of the AAAI Conference on Artificial Intelligence, volume 33, pages 6618-6625, 2019.

[14] X. Kong, Q. Xie, Z. Dai, and E. Hovy. Fast and simple mixture of softmaxes with bpe and hybrid-lightrnn for language generation. In Proceedings of the AAAI Conference on Artificial Intelligence, volume 33, pages 6626-6633, 2019.

[15] X. Kong, X. Yang, M. Hasegawa-Johnson, J.-Y. Choi, and S. Shattuck-Hufnagel. Landmarkbased consonant voicing detection on multilingual corpora. arXiv preprint arXiv:1611.03533, 2016.

[16] M. P. Kumar, B. Packer, and D. Koller. Self-paced learning for latent variable models. In Advances in Neural Information Processing Systems, pages 1189-1197, 2010.

[17] Y. J. Lee and K. Grauman. Learning the easy things first: Self-paced visual category discovery. In Computer Vision and Pattern Recognition (CVPR), 2011 IEEE Conference on, pages 17211728. IEEE, 2011.

[18] J. Liu and X. Zhu. The teaching dimension of linear learners. Journal of Machine Learning Research, 17(162):1-25, 2016. 
[19] W. Liu, B. Dai, J. M. Rehg, and L. Song. Iterative machine teaching. arXiv preprint arXiv:1705.10470, 2017.

[20] A. Santoro, S. Bartunov, M. Botvinick, D. Wierstra, and T. Lillicrap. Meta-learning with memory-augmented neural networks. In International conference on machine learning, pages $1842-1850,2016$.

[21] J. Suh, X. Zhu, and S. Amershi. The label complexity of mixed-initiative classifier training. In International Conference on Machine Learning, pages 2800-2809, 2016.

[22] J. S. Supancic III and D. Ramanan. Self-paced learning for long-term tracking. In Computer Vision and Pattern Recognition (CVPR), 2013 IEEE Conference on, pages 2379-2386. IEEE, 2013.

[23] I. Sutskever, J. Martens, G. Dahl, and G. Hinton. On the importance of initialization and momentum in deep learning. In International conference on machine learning, pages 11391147, 2013.

[24] Y. Tsvetkov, M. Faruqui, W. Ling, B. MacWhinney, and C. Dyer. Learning the curriculum with bayesian optimization for task-specific word representation learning. arXiv preprint arXiv:1605.03852, 2016.

[25] R. J. Williams. Simple statistical gradient-following algorithms for connectionist reinforcement learning. In Reinforcement Learning, pages 5-32. Springer, 1992.

[26] M. Xia, X. Kong, A. Anastasopoulos, and G. Neubig. Generalized data augmentation for low-resource translation. In Proceedings of the 57th Annual Meeting of the Association for Computational Linguistics, pages 5786-5796, 2019.

[27] F. Yang, T. Fei, Q. Tao, L. Xiang-yang, and L. Tie-yan. Learning to teach. In Proceedings of the International Conference on Learning Representations (ICLR), 2018.

[28] X. Zhu. Machine teaching for bayesian learners in the exponential family. In Advances in Neural Information Processing Systems, pages 1905-1913, 2013.

[29] X. Zhu. Machine teaching: An inverse problem to machine learning and an approach toward optimal education. In $A A A I$, pages 4083-4087, 2015. 